\title{
Seismic Rays as Finsler Geodesics
}

\author{
Peter L. Antonelli* Andrej Bóna ${ }^{\dagger} \quad$ Michael A. Slawiński ${ }^{\ddagger}$
}

July 19, 2010

\begin{abstract}
We prove that, in general, for anisotropic nonuniform continua, seismic rays are geodesics in Finsler geometry. In particular, for separable velocity functions, the geometry is Wagnerian. We provide concrete examples with theoretical discussions and introduce the seismic Finsler metric.
\end{abstract}

\section{Introduction}

In seismological studies one often measures traveltime of a signal propagating between a source and a receiver. It is common to think of a signal propagation in terms of rays. Seismic ray theory has been discussed extensively in the literature. For instance, the books of Achenbach (1973), Červený (2001), Achenbach et al. (1982), and Kravtsov and Orlov (1990) constitute an important contributions to this field. Rays can be obtained either by solving the elastodynamic equations with the method of characteristics or by considering a generalization of Fermat's variational principle. It can be shown that these two approaches are almost equivalent (Epstein and Śniatycki, 1992).

In view of Fermat's variational principle, rays are geodesics in a certain geometry. Since rays are not, in general, straight lines, the geometry in question is not the Euclidean geometry of physical space. In this paper, we discuss the relevance of the geometry of Fermat's principle, which we show to be a Finsler geometry. Recent developments in Finsler geometry have been described by Antonelli et al. (1993), Bao et al. (2000), Miron and Anastasiei (1994), and Shen (2001).

\section{Concept of a ray}

Fundamentally, rays are mathematical entities resulting from the solution of the eikonal equation by the method of characteristics. The eikonal equation arises

\footnotetext{
*Department of Mathematical and Statistical Sciences, University of Alberta, Canada, pa2@pop.srv.ualberta.ca

$\dagger$ Department of Mathematics and Statistics, University of Calgary, Canada, bona@math.ucalgary.ca

${ }_{\ddagger}^{\ddagger}$ Department of Earth Sciences, Memorial University of Newfoundland, Canada, mslawins@mun.ca
} 
by considering the elastodynamic equations, which are the Cauchy equations of motion - namely ${ }^{1}$,

$$
\rho(x) \frac{d^{2} u^{i}}{d t^{2}}=\frac{\partial \sigma^{i j}}{\partial x^{j}}
$$

where $\rho(x)$ is the mass density at point $x, u$ is a vector describing the displacement of the continuum, and $\sigma_{i j}$ is the stress tensor - in the context of linear elasticity, where stress is given by

$$
\sigma^{i j}=c^{i j k l}(x) \varepsilon_{k l},
$$

with $c^{i j k l}(x)$ denoting the elasticity constants at point $x$, and $\varepsilon_{k l}$ being the strain tensor.

If we assume the solution of the elastodynamic equations to be of the form

$$
u(x, t)=A(x) f(\psi(x)-t),
$$

where the level sets of $\psi$ correspond to wavefronts, the Cauchy equations of motion become

$$
\left\{\begin{array}{l}
\frac{\partial c^{i j k l}(x)}{\partial x^{j}} \frac{\partial A_{k}}{\partial x^{l}}+c^{i j k l}(x) \frac{\partial^{2} A_{k}}{\partial x^{j} \partial x^{l}}=0 \\
\frac{\partial}{\partial x^{j}}\left(c^{i j k l}(x) A_{l} \frac{\partial \psi}{\partial x^{k}}\right)+c^{i j k l}(x) \frac{\partial A_{k}}{\partial x^{l}} \frac{\partial \psi}{\partial x^{j}}=0 \\
c^{i j k l}(x) A_{k} \frac{\partial \psi}{\partial x^{j}} \frac{\partial \psi}{\partial x^{l}}-\rho(x) A_{i}=0
\end{array} .\right.
$$

The condition for existence of nontrivial solutions for the last equation of the set (1) can be stated as

$$
\operatorname{det}\left[c^{i j k l}(x) \frac{\partial \psi}{\partial x^{j}} \frac{\partial \psi}{\partial x^{l}}-\rho(x) \delta^{i k}\right]=0 .
$$

Denoting

$$
p_{j}:=\frac{\partial \psi}{\partial x^{j}}
$$

we obtain a third-degree polynomial in $p^{2}$, which can be written as

$$
\left(p^{2}-\frac{1}{v_{1}^{2}\left(x, \frac{p}{|p|}\right)}\right)\left(p^{2}-\frac{1}{v_{2}^{2}\left(x, \frac{p}{|p|}\right)}\right)\left(p^{2}-\frac{1}{v_{3}^{2}\left(x, \frac{p}{|p|}\right)}\right)=0 .
$$

Herein, the quantities $1 / v_{i}^{2}(x, p /|p|)$ are the roots of the polynomial and, in general, correspond to the three phase slownesses of the threes wave types that exist in anisotropic continua. Each factor of expression (3) is the eikonal equation for a given wave type, namely,

$$
p^{2}=\frac{1}{v^{2}\left(x, \frac{p}{|p|}\right)} \text {. }
$$

\footnotetext{
${ }^{1}$ In this paper we use the Einstein summation convention.
} 
Equation (4) is the key equation of ray theory. This equation states that the slowness of the wavefront propagation - given by $p^{2}=p_{i} p^{i}$, where, as defined in expression (2), $p=\nabla \psi$ is a vector normal to the wavefront - is a function of the properties of the continuum, $v(x, p /|p|)$, which depend on position and direction, respectively. The function $v(x, p /|p|)$ is called the phasevelocity function. In a mathematical context, equation (4) belongs to the class of the Hamilton-Jacobi equations.

To solve the eikonal equation we use the method of characteristics. Setting the Hamiltonian $H$ to be

$$
H(x, p)=\frac{1}{2} p^{2} v^{2}\left(x, \frac{p}{|p|}\right)
$$

the equations of the characteristics are the Hamilton ray equations, given by

$$
\left\{\begin{array}{l}
\dot{x}^{i}:=\frac{d x^{i}}{d t}=\frac{\partial H}{\partial p_{i}} \\
\dot{p}_{j}:=\frac{d p_{j}}{d t}=-\frac{\partial H}{\partial x^{j}}
\end{array},\right.
$$

where the factor of $1 / 2$ in the Hamiltonian, defined in expression (5), comes from the parametrization of the rays by the physical time.

Considering the Hamiltonian to be regular, which is always the case for the fastest wave in a linearly elastic continuum (Musgrave, 1970), one can use the Legendre transformation to state the raytracing problem in the context of the calculus of variations, namely,

$$
\delta \int L d t=0
$$

where the Lagrangian is

$$
L=p_{i} \dot{x}^{i}-H \text {. }
$$

Hence, rays satisfy the Euler equation ${ }^{2}$

$$
\frac{\partial L}{\partial x^{i}}-\frac{d}{d t}\left(\frac{\partial L}{\partial \dot{x}^{i}}\right)=0 .
$$

This variational problem is, however, parameter dependent. To obtain a parameter independent variational problem one must consider a function $F(x, \dot{x})$ that is homogeneous of degree one in the $\dot{x}^{i}$ and yields the same solutions as $L$.

Considering expression (5) and the fact that, for physical reasons, the phasevelocity function, $v(x, p /|p|)$, is homogeneous of degree zero in the $p_{i}$, we conclude that $H\left(x_{i}, p_{i}\right)$ is homogeneous of degree two in the $p_{i}$. Since $H\left(x_{i}, p_{i}\right)$ is homogeneous of degree two in the $p_{i}$, then

$$
L\left[x_{i}, \dot{x}_{i}\left(x_{i}, p_{i}\right)\right]=H\left(x_{i}, p_{i}\right),
$$

where, by the inverse Legendre transformation, $\dot{x}_{i}=\partial H / \partial p_{i}$. Moreover, the Lagrangian, $L\left(x_{i}, \dot{x}_{i}\right)$, is homogeneous of degree two in the $\dot{x}_{i}$. For this reason

\footnotetext{
${ }^{2}$ These equations are also commonly referred to as the Euler-Lagrange equations.
} 
a natural candidate for the function $F$ is given by $L=F^{2} / 2$. It is easy to show that, if $d F / d t=0$ and if $F \neq 0$, then if $x(t)$ is a solution of Euler equations (6), becomes the then $\mathbf{x}(t)$ is also the solution of the Euler equations

$$
\frac{\partial F}{\partial x^{i}}-\frac{d}{d t}\left(\frac{\partial F}{\partial \dot{x}^{i}}\right)=0 .
$$

The fact that $F$ yields the same solutions as $L$ and is homogeneous of degree one in the $\dot{x}^{i}$ makes it the proper choice to state Fermat's principle of stationary traveltime as

$$
\delta \int F d t=0
$$

\section{Finsler geometry}

\subsection{Geodesics and connections}

Euler equations (7) suggest studying a geometry for which the rays are geodesics. To find such a geometry let $M^{n}$ denote a closed, connected, $C^{\infty}$-manifold and $T M^{n}$ its tangent bundle with 0 -section removed. Let

$$
F: T M^{n} \rightarrow \mathbb{R}
$$

be a $C^{\infty}$ function (positively)-homogeneous of degree one in $y^{i}=\dot{x}^{i}, i=$ $1,2, \ldots, n$.

If the Hessian matrix

$$
g_{i j}(x, y)=\dot{\partial}_{i} \dot{\partial}_{j}\left(\frac{1}{2} F^{2}\right)
$$

of second partial derivatives with respect to $y^{i}$ and $y^{j}$ (or, what is the same, $d x^{i} / d t=\dot{x}^{i}$ and $\left.d x^{j} / d t=\dot{x}^{j}\right)$ is non-singular in some open conical subset $\mathbb{D} \subseteq T M^{n}$, then the Euler equations are equivalent to the geodesic equations

$$
\frac{d^{2} x^{i}}{d t^{2}}+\gamma_{j k}^{i}(x, y) \frac{d x^{j}}{d t} \frac{d x^{k}}{d t}=0, \quad i=1, \ldots, n
$$

where

$$
\gamma_{j k}^{i}(x, y)=\frac{1}{2} g^{i r}\left(\partial_{k} g_{r j}+\partial_{j} g_{r k}-\partial_{r} g_{j k}\right)
$$

are the so-called Christoffel symbols of 2 nd kind. Here $t$ is traveltime and

$$
(d t)^{2}=F^{2}(x, d x)=g_{i j}(x, y) d x^{i} d x^{j}
$$

and $g^{i \ell} g_{\ell k}=\delta_{k}^{i}$, so that $\left(g^{i j}\right)$ is the inverse of $\left(g_{i j}\right)$, and $\partial_{k}$ is the partial with respect to $x^{k}$. Moreover, upon non-singular coordinate transformation $x^{i} \rightarrow \bar{x}^{i}$, and the induced transformation $y^{i} \rightarrow \bar{y}^{i}$, by the Jacobian, $g_{i j}(x, y)$ transforms as a Finsler tensor covariant of rank 2, which is to say it transforms as in classical tensor analysis (this is true of all Finsler tensors regardless of type). 
We remark that $F(x, d x / d t)$ is conserved along geodesics. It has value one and defines the indicatrix surface at each point $x$. Introduce the unit length element of support $\ell^{i}=y^{i} / F$ and the angular metric tensor

$$
h_{i j}=g_{i j}-\ell_{i} \ell_{j}
$$

where $\ell_{i}=g_{i r} \ell^{r} . h_{i j}$ is the induced metric tensor defined on the indicatrix surface. It is globally defined on the indicatrix subbundle of the slit tangent bundle $T M^{n}$, just as $g_{i j}$ is globally defined on $T M^{n}$. Another important Finsler object is the Cartan torsion tensor.

$$
C_{i j k}=\frac{1}{2} \dot{\partial}_{k} g_{i j}(x, y)
$$

from where we get

$$
V_{j k}^{i}:=C_{j k}^{i}:=g^{i r} C_{j r k},
$$

which defines a vertical connection, that is, a vertical covariant differentiation $\left(\nabla^{v}\right)$. For example, for any tensor $A_{j}^{i}(x, y)$,

$$
\nabla_{k}^{v} A_{j}^{i}:=\dot{\partial}_{k} A_{j}^{i}+A_{j}^{r} C_{r k}^{i}-A_{r}^{i} C_{j k}^{r}
$$

We remark that $C_{i j k}=0$ if and only if $g_{i j}$ is Riemannian (i.e., independent of the $\left.\dot{x}^{i}\right)$.

Using the geodesic equations (10) in the form

$$
\frac{d^{2} x^{i}}{d t^{2}}+2 G^{i}\left(x, \frac{d x}{d t}\right)=0, \quad i=1, \ldots, n
$$

we define the nonlinear Berwald connection coefficients of $\left(M^{n}, F\right)$ by

$$
G_{j}^{i}:=\dot{\partial}_{j} G^{i}
$$

and introduce the Berwald gradient operator $\delta_{i}=\partial_{i}-G_{i}^{r} \dot{\partial}_{r}$. The horizontal and vertical Berwald connection coefficients are defined by

$$
G_{j k}^{i}:=\dot{\partial}_{k} G_{j}^{i}, \quad \text { and } \quad V_{j k}^{i}=0
$$

in (16). From $(17)_{b, c}$, we define the horizontal covariant differentiation $\nabla^{h}$, for example,

$$
\nabla_{k}^{h} A_{j}^{i}:=\partial_{k} A_{j}^{i}-\left(\dot{\partial}_{r} A_{j}^{i}\right) G_{k}^{r}+A_{j}^{r} G_{r k}^{i}-A_{r}^{i} G_{j k}^{r} .
$$

The Ricci identities are given by the usual commutation relations

$$
\begin{aligned}
\nabla_{k}^{h} \nabla_{s}^{h} A_{j}^{i}-\nabla_{s}^{h} \nabla_{k}^{h} A_{j}^{i} & =A_{j}^{r} G_{r s k}^{i}-A_{r}^{i} G_{j s k}^{r}-\left(\dot{\partial}_{r} A_{j}^{i}\right) \mathbb{R}_{s k}^{r}, \\
\nabla_{k}^{h} \nabla_{s}^{v} A_{j}^{i}-\nabla_{s}^{v} \nabla_{k}^{h} A_{j}^{i} & =A_{j}^{r} \mathbb{D}_{r s k}^{i}-A_{r}^{i} \mathbb{D}_{j s k}^{r},
\end{aligned}
$$

where

$$
G_{j s k}^{i}=\delta_{k} G_{j s}^{i}+G_{j s}^{r} G_{r k}^{i}-\delta_{s} G_{j k}^{i}-G_{j k}^{r} G_{r s}^{i}
$$


is so-called (h) h-curvature and

$$
\mathbb{D}_{j h k}^{i}:=\dot{\partial}_{k} G_{j h}^{i}
$$

which detects angular dependence in the connection $G_{j k}^{i}$, is the Douglas tensor (or (v) h-curvature of the Berwald connection), while

$$
\mathbb{R}_{h k}^{i}=\partial_{k} G_{h}^{i}-G_{k}^{r} G_{h r}^{i}-\partial_{h} G_{k}^{i}+G_{h}^{r} G_{k r}^{i}
$$

is the so-called (v) h-torsion. See Antonelli et al. for explanation of the prefixes. We remark that geodesics are Euclidean straight lines if and only if $\mathbb{D}=0=$ $\mathbb{R}$ in (18) and (19).

We now turn our attention to another important connection called the Cartan connection $\mathbb{C} \Gamma=\left(\Gamma_{j k}^{i}, G_{j}^{i}, C_{j k}^{i}\right)$ of $\left(M^{n}, F\right) .{ }^{3}$ It is characterized by five axioms, namely,

1. $\nabla_{k}^{h} g_{i j}=0 \quad(h$-metrical $)$

2. $\nabla_{k}^{v} g_{i j}=0 \quad(v$-metrical $)$

3. $S_{j k}^{i}:=C_{j k}^{i}-C_{k j}^{i}=0 \quad(v$ - symmetric $)$

4. $T_{j k}^{i}:=\Gamma_{j k}^{i}-\Gamma_{k j}^{i}=0 \quad(h$-symmetric $)$

5. $D_{j}^{i}=y^{r} \Gamma_{r j}^{i}-G_{j}^{i}=0$ (deflection tensor $D$ vanishes)

Note that axiom 3 is superfluous in our development here because we defined the vertical covariant derivative in terms of the tensor of Cartan (15). Had we used a general tensor $V_{j k}^{i}$, then axiom 3 would have been necessary to secure $\Gamma_{j k}^{i}$ as the coefficients of the Cartan connection. Note that $\delta_{i} f$ is a covariant Finsler vector field, while, in general, $\partial_{i} f$ is not when $f$ is a smooth function on $T M^{n}$. Of course, if $f$ has no $y$-dependence then $\partial_{i} f$ is a vector.

We now have the following theorem.

Theorem 1 The (horizontal) Cartan connection coefficients are given locally by (11) with $\partial_{k}$ being replaced by $\delta_{k}$.

Using the triple notation we have $\mathbb{C} \Gamma=\left(\Gamma_{j k}^{i}, G_{j}^{i}, C_{j k}^{i}\right)$ for the Cartan connection $\mathbb{C} \Gamma$ and $\mathbb{B} \Gamma=\left(G_{j k}^{i}, G_{j}^{i}, 0\right)$ for the Berwald connection $\mathbb{B} \Gamma$. Thus, (16) is the vertical covariant derivative of $A_{j}^{i}$ according to the Cartan connection $\mathbb{C} \Gamma$ while

$$
{ }_{B} \nabla_{k}^{v} A_{j}^{i}:=\dot{\partial}_{k} A_{j}^{i}
$$

gives it for the Berwald connection $\mathbb{B} \Gamma$ (the missing term, compared to (17) , $_{\text {, }}$ explains the zero in the third slot of the Berwald triple). We remark that if there are coordinates $\bar{x}$ for which $F$ is independent of $\bar{x}$, then $(17)_{a}$ has $G^{i} \equiv 0$.

\footnotetext{
${ }^{3}$ Here we use the "triple" notation of Matsumoto, (Antonelli et al.).
} 
Such a space is called locally Minkowski. However, $C_{i j k}$ are not generally zero even in this case.

Both the above connections are important in Finsler geometry. The Cartan $\mathbb{C} \Gamma$ is defined entirely in terms of the metric function $F$ and its derivatives. The Berwald connection $\mathbb{B} \Gamma$ comes directly from the geodesic equations of $\left(M^{n}, F\right)$. However, the Berwald connection satisfies

$$
{ }_{B} \nabla_{k}^{h} g_{i j}=-2_{B} \nabla_{l}^{h} C_{i j k} y^{l} .
$$

This expression is generally not zero! If we replace ${ }_{B} \nabla$ (Berwald) by $\nabla$ (Cartan) in (21), the left side must be equal to zero. This is the so-called $h$-Ricci lemma. In fact, both $h$ and $v$-Ricci lemmas hold for $\mathbb{C} \Gamma$ and both fail for $\mathbb{B} \Gamma$. For the wellknown axiomatic characterization of the Berwald connection and more detail on that of Cartan (Antonelli et al., 1993).

We wish to consider yet another connection, called the Wagner connection, $W \Gamma$. A Wagner connection $W \Gamma$ on $\left(M^{n}, F\right)$ is similar to the Cartan connection in that the above axioms are the same except for the axiom 4, which is replaced by $4^{\prime}$,

$$
\tau_{j k}^{i}=T_{j k}^{i}-\frac{1}{n+1} \delta_{j}^{i} T_{a k}^{a}-\frac{1}{n+1} \delta_{k}^{i} T_{j a}^{a}=0,
$$

where $\tau_{j k}^{i}$ is called the Thomas' tensor (J.M. Thomas). The vanishing of Thomas' tensor is equivalent to the existence of a covariant field $\sigma_{i}(x, y)$ such that

$$
T_{j k}^{i}=\delta_{j}^{i} \sigma_{k}-\delta_{k}^{i} \sigma_{j} .
$$

In the classical literature the Wagner connection is said to have semi-symmetric torsion. To link the Wagner geometry with seismology let us recall the following theorem.

Theorem 2 (Hashiguchi) A Finsler space $\left(M^{n}, F\right)$ is conformal to a locally Minkowski space if and only if there exists a Wagner connection $W \Gamma=$ $\left(F_{j k}^{i}, G_{j}^{i}, C_{j k}^{i}\right)$ on $\left(M^{n}, F\right)$ such that $F_{j k}^{i}$ depends at most on $x^{i}, \sigma_{i}(x)=\partial_{i} \sigma(x)$, and the $h$-curvature of $W \Gamma$ vanishes.

Note that many spaces are conformally Minkowski but by no means all of them, even in dimension two! But let us recall that every 2 dimensional Riemannian space is conformally Euclidean. Note also that the vertical Wagner connection is identical to that of the Cartan connection.

\subsection{Separable velocity function}

In anisotropic nonuniform continua, we often describe the signal velocity along the raypath by a separable velocity function of the form

$$
V(x, \dot{x})=f(x) \phi(\dot{x})
$$


and consider Lagrangians of the form

$$
F(x, \dot{x})=\frac{\sqrt{\dot{x}_{i} \dot{x}^{i}}}{V(x, \dot{x})} .
$$

In expression (23), $f$ characterizes the nonuniformity while $\phi$ characterizes the anisotropy.Following Huygens' formulation, the function $\phi$ can be associated with an elementary wavefront at any given location within a continuum. Theorem 2 gives necessary and sufficient conditions for separability.

\subsection{Two-dimensional Finsler spaces}

We now turn our attention to $n=2$. In this two-dimensional case we assume $g_{i j}$ to be positive-definite on an open conical region of $T M^{n}$. We have, in addition to the unit element of support $\ell^{i}$, a vector field $m^{i}$ such that $g_{i j} m^{i} m^{j}=1$, $g_{i j} m^{i} \ell^{j}=0$ and $h_{i j}=m_{i} m_{j}$ (see equation (13)), where $m_{i}=g_{i r} m^{r}$ and, finally,

$$
g_{i j}=\ell_{i} \ell_{j}+m_{i} m_{j} .
$$

The pair $\left(\ell^{i}, m^{i}\right)$ is the so-called Berwald frame of $\left(M^{2}, F\right)$. Using $C_{i j k} \ell^{k}=0$, one can show that

$$
F C_{i j k}=I m_{i} m_{j} m_{k}
$$

where $I(x, y)$ is the main scalar of $\left(M^{2}, F\right)$. The sign of $I$ depends on the orientation of $m^{i}$ (there are two choices), but $I^{2}$ does not. $I$ is analogous to the eccentricity of an ellipse for Riemannian indicatrices.

Let $g$ denote the determinant of the matrix tensor $g_{i j}$. It is well known that $\sqrt{g} d v$ is the elemental volume form, such that, area of $\Omega \subseteq T M^{n}$ is given by

$$
\int_{\Omega} \sqrt{g} d V
$$

Letting $\vartheta$ be the arclength along the indicatrix at a point $P$, the formula (Antonelli et al., 1993)

$$
\partial_{\vartheta} \sqrt{g}=I \sqrt{g}
$$

or

$$
\partial_{\vartheta} \ln \sqrt{g}=I
$$

can be interpreted as a rate of change, relative to $\vartheta$, of the solid angle of rays intersecting the elementary wavefront $F_{p}=1$.

Furthermore, Berwald's classical result

$$
\mathbb{R}_{j k}^{i}=F \mathbb{R} m^{i}\left(\ell_{j} m_{k}-\ell_{k} m_{j}\right),
$$

where $\mathbb{R}(x, y)$ is Berwald's Gauss Curvature Scalar is very important. It is wellknown that $I$ and $\mathbb{R}$ determine the geometry of $\left(M^{2}, F\right)$ up to local isometry (Antonelli et al., 1993.).

$\mathbb{R}$ determines the spreading $(\mathbb{R} \leq 0)$ and focusing $(\mathbb{R}>0)$ of the Finsler geodesics. In a geophysical context, if the ray velocity increases with the depth 
and the indicatrices (elementary wavefronts) are convex, the rays are spreading so the curvature $\mathbb{R}$ is expected to be non-positive. For nonconvex elementary wavefronts one can encounter focusing of the rays, which leads to caustics (e.g., Hanyga and Slawinski, 2000).

\subsection{Berwald spaces and Wagner spaces}

The simplest kind of Finsler spaces beyond the locally Minkowski (whose tangent planes are curved for $n \geq 3$, in general) are the Berwald spaces. These are characterized by

or, equivalently, by

$$
{ }_{C} \nabla_{\ell}^{h} C_{i j k}=0
$$

$$
{ }_{B} \nabla_{\ell}^{h} C_{i j k}=0 \text {. }
$$

For $n=2$ a complete isometric classification was given by L. Berwald (see Antonelli et al., 1993). All of these two-dimensional Berwald spaces which are not locally Minkowski have main scalar $I$ equal to a constant. Of these, exactly four classes are distinguished, three are positive-definite with $I^{2}<4, I^{2}=4$, $I^{2}>4$, (Antonelli et al., 1993). In this classic case $\mathbb{R}=0$.

Wagner spaces of dimension $n$ are by definition Finsler spaces which have a Wagner connection with its $\sigma_{i}$-field being a gradient, $\sigma_{i}(x)=\partial_{i} \sigma(x)$. They are generalizations of Berwald spaces in many respects. A notable example of this relationship is the

Theorem 3 A. $\left(M^{n}, F\right)$ is $\sigma$-Wagner if and only if

$$
{ }_{W} \nabla_{\ell}^{h} C_{i j k}=0 \text {. }
$$

B. $\left(M^{n}, F\right)$ is Berwald if and only if $(43)_{b}$ holds.

All Berwald spaces are trivial (i.e., $\sigma_{i} \equiv 0$ ) examples of Wagner spaces. From Theorem 2, we can start with any locally Minkowski space $\left(M^{n}, F\right)$ and form a Wagner space by using $F=e^{\sigma(x)} \cdot \bar{F}$ in $M^{n}$. This Wagner space $\left(M^{n}, F\right)$ has a linear (affine) connection $F_{j k}^{i}(x)$ and its (usual) curvature tensor is just the horizontal Wagner curvature, which vanishes. It is notable that the geodesics of $\left(M^{n}, \bar{F}\right)$ are never, for $\sigma_{i} \neq 0$, the autoparallels

$$
\frac{d^{2} x^{i}}{d t^{2}}+F_{j k}^{i}(x) \frac{d x^{j}}{d t} \frac{d x^{k}}{d t}=0
$$

of $W \Gamma$ (Antonelli et al., 1993). As an example, let us write

$$
F\left(z, \frac{\dot{x}}{\dot{z}}\right)=e^{\sigma(z)} \cdot \frac{\sqrt{(\dot{x})^{2}+(\dot{z})^{2}}}{\phi\left(\frac{\dot{x}}{\dot{z}}\right)}=e^{\sigma(z)} \cdot \bar{F}\left(\frac{\dot{x}}{\dot{z}}\right)
$$

with $\sigma(z)=-\ln f(z)$. Here $x^{1}=x, x^{2}=z, \dot{x}^{1}=\dot{x}, \dot{x}^{2}=\dot{z}$, and $\bar{F}$, the anisotropic part, is a Finsler function of a Minkowski space. The Wagner autoparallels are solutions of

$$
\frac{d^{2} x^{i}}{d t^{2}}+\left(\delta_{j}^{i} \sigma_{k}\right) \frac{d x^{j}}{d t} \frac{d x^{k}}{d t}=0
$$


where $\sigma_{1}=\partial_{1} \sigma=\partial_{x} \sigma, \sigma_{2}=\partial_{2} \sigma(z)=\partial_{z} \sigma(z)$, whereas the geodesics are the solutions of

$$
\frac{d^{2} x^{i}}{d t^{2}}+\left(\delta_{j}^{i} \sigma_{k}\right) \frac{d x^{j}}{d t} \frac{d x^{k}}{d t}=\mathcal{K}^{i},
$$

where

$$
\mathcal{K}^{i}=\bar{F}^{2} \bar{g}^{i j} \sigma_{j}-\delta_{j}^{i} \sigma_{k} \frac{d x^{j}}{d t} \frac{d x^{k}}{d t},
$$

with $\bar{g}^{i j}$ being the inverse of

$$
\bar{g}_{i j}=\dot{\partial}_{i} \dot{\partial}_{j}\left(\frac{1}{2} \bar{F}^{2}\right) .
$$

Moreover, $\mathcal{K}^{i}$ is orthogonal to $d x^{j} / d t$, namely,

$$
\bar{g}_{i j} \mathcal{K}^{i} \frac{d x^{j}}{d t}=0,
$$

so that $\mathcal{K}^{i}$ is Wagner curvature of a solution of $(32)_{a, b}$. This means that geodesics are curved in Wagner geometry and $\mathcal{K}^{i}$ measures that "curvature". Of course, geodesics are not curved in their usual geometry.

Let us take a specific form for $\phi(\dot{x} / \dot{z})$ above, say

$$
\phi\left(\frac{\dot{x}}{\dot{z}}\right)=\frac{\left[(\dot{x})^{2}+(\dot{z})^{2}\right]^{1 / 2}}{\left[(\dot{x})^{m}+(\dot{z})^{m}\right]^{1 / m}} .
$$

where $m$ is an even integer $\geq 2$. Furthermore, let us take the linear form $\left(\alpha_{1}\right.$, $\alpha_{2}$ positive constants)

$$
\sigma=-\ln f(x, z)=-\ln \left(\alpha_{1}+\alpha_{2} z\right)
$$

to allow $z$ dependence. We then obtain the Finsler space $\left(M^{2}, F\right)$ called the seismic Finsler space with metric function

$$
F\left(x, z, \frac{\dot{x}}{\dot{z}}\right)=e^{-\ln f} \cdot\left[(\dot{x})^{m}+(\dot{z})^{m}\right]^{1 / m},
$$

where

$$
I=\frac{m-2}{2 \sqrt{m-1}}\left(\frac{1-\left(\frac{\dot{z}}{\dot{x}}\right)^{m}}{\sqrt{\left(\frac{\dot{z}}{\dot{x}}\right)^{m}}}\right) .
$$

The Berwald Gauss curvature scalar $\mathbb{R}$ of this space is

$$
\mathbb{R}=\frac{\frac{1}{4} m b^{2}(\dot{z})^{2-2 m} \cdot\left[(m-2) \cdot(\dot{x})^{m}-m \cdot(\dot{z})^{m}\right]}{(m-1)^{2}\left((\dot{x})^{m}+(\dot{z})^{m}\right)^{\frac{2-m}{m}}} .
$$

From this, we see that for $m>0, b>0$, the ray angle $\theta$ determines where the curvature vanishes

$$
\mathbb{R}=0 \Longleftrightarrow \frac{\dot{x}}{\dot{z}}:=\tan \theta=\frac{1}{\left(1-\frac{2}{m}\right)^{1 / m}} .
$$


Ray spreading $(\mathbb{R} \leq 0)$ occurs when

$$
\tan \theta \leq \frac{1}{\left(1-\frac{2}{m}\right)^{1 / m}} .
$$

The boundary of the $(m>2)$-case is a straight line whose slope is $\tan \theta$, and as $m \rightarrow+\infty$, this $\theta$ approaches $\pi / 4$. The negatively curved region is between the above line and the $\dot{z}$-axis. The case $m=2$ is Riemannian and $\mathbb{R}=-b^{2}$, a negative constant and there is no boundary. The region of positive curvature (ray focusing) arises from $\dot{z}$ being very small. For $\dot{z}$ small we expect "surface effects".

Finally, the Douglas tensor $\mathbb{D}_{j k l}^{i}$ is never zero if $m>2$ and vanishes for $m=2$. This means the connection coefficients of the ray paths, $G_{j k}^{i}$, depend on the ray angle, $\theta$, if and only if $m>2$.

\section{Conclusions and future work}

In general, for propagation of seismic signals in anisotropic, nonuniform continua, raypaths are geodesics of a Finsler geometry. In such a case, velocity along the raypath is a function of both direction and position. Also, in seismic investigations, we often use a velocity function that is separable. In other words, anisotropy and nonuniformity are described by a product of two functions that correspond to the angular and spatial dependence, respectively. In such a case the subset of the Finsler geometry in question is the Wagner geometry.

The study of seismic raypaths in terms of a Finsler geometry allows us to investigate these mathematical entities in their natural mathematical context as geodesics. Furthermore, Finsler geometry has been intensely studied over last quarter of a century and provides a fruitful platform for convenient formulations of physical problems, Antonelli et al. (1993), Bao et al. (2000), Miron and Anastasiei (1994), and Shen (2001).

\section{Acknowledgements}

We wish to thank Drs. I. Bucataru and S. Rutz for fruitful discussions and the latter for also helping with computing the expressions and graphical behaviour of some geometrical objects for the example in Section 3 by means of the Finsler computer package [Rutz and Portugal]. Thanks also to Vivian Spak for her excellent typesetting.

This study has been done in the context The Geomechanics Project. Hence, the authors wish to acknowledge the support and encouragement of PanCanadian Energy and Talisman Energy. 


\section{References}

Achenbach, J.D., 1973. Wave propagation in elastic solids: North Holland.

Achenbach, J.D., Gausten, A.K., and McMaken, H., 1982. Ray methods for waves in elastic solids: with applications to scattering by cracks: Pitman Publishing Inc.

Antonelli, P.L., Ingarden, R.S. and Matsumoto, M., 1993. The theory of sprays and Finsler spaces with application in Physics and Biology: Kluwer Academic Publishers, Dordrecht-Boston-London.

Bao, D., Chern, S.-S., and Shen, Z., 2000. An introduction to RiemannFinsler geometry: Springer-Verlag.

Červený, V., 2001. Seismic ray theory: Cambridge University Press

Epstein, M., Śniatycki, J., 1992. Fermat's principle in elastodynamics. Journal of Elasticity, 27, $45-56$.

Hanyga, A., and Slawinski, M.A., 2000. Caustics in qSV rayfields of transversally isotropic and vertically inhomogeneous media: SEG (Special Issue).

Kravtsov, Y.A., and Orlov, Y.I., 1990. Geometrical optics of inhomogeneous media: Springer-Verlag.

Miron, R., and Anastasiei, M., 1994. The geometry of Lagrange spaces: theory and applications: 59, Fund. Theor. Phys. Series, Kluwer Academic Publishers, Dordrecht-Boston-London.

Musgrave, M.J.P., 1970. Crystal acoustics: Introduction to the study of elastic waves and vibrations in crystals: Holden-Day.

Rutz, S.F. and Portugal, R., 2001. Finsler: A computer algebra package for Finsler geometry, Nonlinear Analysis 47, 6121-6134. Proceedings of the World Congress of Nonlinear Analysts (WCNA 2000) Catania, Sicily,Italy, 19 26 July 2000.

Shen, Z., 2001. Differential geometry of spray and Finsler spaces: Kluwer Academic Publishers, Dordrecht-Boston-London. 